Gut and Liver, Vol. 10, No. 1, January 2016, pp. 83-94

\title{
Room for Quality Improvement in Endoscopist-Directed Sedation: Results from the First Nationwide Survey in Korea
}

Chang Kyun Lee*, Seok Ho Dong*, Eun Sun Kim", Sung-Hoon Moon ${ }^{\ddagger}$, Hong Jun Park ${ }^{\S}$, Dong-Hoon Yang", Young Chul Yoo", Tae Hoon Lee ${ }^{\#}$, Sang Kil Lee**, Jong Jin Hyun ${ }^{\dagger}$, and The Korean Society of Gastrointestinal Endoscopy Task Force on Endoscopic Sedation

"Department of Internal Medicine, Kyung Hee University School of Medicine, ${ }^{\dagger}$ Department of Internal Medicine, Korea University College of Medicine, Seoul, ${ }^{\ddagger}$ Department of Internal Medicine, Hallym University College of Medicine, Anyang, ${ }^{s}$ Department of Internal Medicine, Yonsei University Wonju College of Medicine, Wonju, "Department of Gastroenterology, University of Ulsan College of Medicine, "Department of Anesthesiology and Pain Medicine, Yonsei University College of Medicine, Seoul, "Department of Internal Medicine, Soonchunhyang University College of Medicine, Cheonan, and "*Department of Internal Medicine, Yonsei University College of Medicine, Seoul, Korea

Background/Aims: This study sought to characterize the current sedation practices of Korean endoscopists in realworld settings. Methods: All active members of the Korean Society of Gastrointestinal Endoscopy were invited to complete an anonymous 35-item questionnaire. Results: The overall response rate was $22.7 \%(1,332 / 5,860)$. Propofolbased sedation was the dominant method used in both elective esophagogastroduodenoscopy (55.6\%) and colonoscopy (52.6\%). The mean satisfaction score for propofol-based sedation was significantly higher than that for standard sedation in both examinations (all $p<0.001$ ). The use of propofol was supervised exclusively by endoscopists (98.6\%). Endoscopists practicing in nonacademic settings, gastroenterologists, or endoscopists with $<10$ years of endoscopic practice were more likely to use propofol than were their counterparts (all $p<0.001$ ). In total, $27.3 \%$ of all respondents performed sedation practices without having undergone sedation training, and $27.4 \%$ did so without any formal sedation protocols. The choice of propofol as the dominant sedation method was the only significant predictor of endoscopist experience with serious sedation-related adverse events (odds ratio, 1.854; 95\% confidence interval, 1.414 to 2.432). Conclusions: Endoscopist-directed propofol administration is the predominant sedation method used in Korea. This survey strongly suggests that there is much room for quality improvement regarding sedation training and patient vigilance in endoscopist-directed sedation. (Gut Liver 2016;10:83-94)

Key Words: Gastrointestinal endoscopy; Sedation; Health care surveys; Propofol; Non-anesthesiologist-administered propofol

\section{INTRODUCTION}

Endoscopic sedation has become an integral part of modern gastrointestinal (GI) endoscopy. It provides better patient satisfaction and compliance with endoscopic procedures; thus, it can facilitate endoscopist performance of safe and effective procedures with patient cooperation. ${ }^{1,2}$ The increasing need for minimally invasive endoscopic procedures and screening endoscopy is also expected to increase the demand for procedural sedation. ${ }^{3,4}$ However, sedation in GI endoscopy is accompanied by several drawbacks, such as additional time for patient recovery, increased healthcare costs, and a substantial risk of cardiopulmonary complications. ${ }^{2,5}$ Additionally, there is continuing debate about who should be responsible for the administration of sedatives, especially propofol. Although increasing evidence has suggested that endoscopist-directed propofol (EDP) can be used safely in elective GI endoscopy for healthy individuals, ${ }^{6-10}$ nonanesthesiologist-administered propofol sedation is still disputed by anesthesia specialists who argue that propofol should be administered only by persons trained in general anesthesia. ${ }^{11-13}$ Moreover, the cost-effectiveness of monitored anesthesia care in low-risk patients has not yet been evaluated sufficiently. ${ }^{14-16}$ Regardless of these continuing debates, the use of EDP is expected to increase steadily worldwide.

Currently, there is wide variation among sedation and concomitant monitoring practices for GI endoscopy, according to

Correspondence to: Chang Kyun Lee

Division of Gastroenterology, Department of Internal Medicine, Kyung Hee University School of Medicine, 26 Kyungheedae-ro, Dongdaemun-gu, Seoul 02447, Korea

Tel: +82-2-958-8258, Fax: +82-2-968-1848, E-mail: cklee92@paran.com Received on July 22, 2015. Revised on September 3, 2015. Accepted on September 4, 2015. pISSN 1976-2283 eISSN 2005-1212 http://dx.doi.org/10.5009/gnl15343

@) This is an Open Access article distributed under the terms of the Creative Commons Attribution Non-Commercial License (http://creativecommons.org/licenses/by-nc/4.0) which permits unrestricted non-commercial use, distribution, and reproduction in any medium, provided the original work is properly cited. 
endoscopists' preferences, resources of the healthcare facility, local policies, and national legal restrictions. ${ }^{17}$ Previous surveys of sedation practice patterns of endoscopists have shown worldwide variation in terms of rates of sedation, preferred sedation regimens, and patient monitoring practices, such as the routine use of pulse oximetry. ${ }^{18-25}$ Despite many studies, some critical questions remain. For example, what are the characteristics of endoscopists who prefer propofol to standard sedation using midazolam and/or opioids? How often do endoscopists follow the quality guidelines for sedation practice, such as preprocedural risk stratification of patients and appropriate training in endoscopic sedation? How many endoscopists have experience with serious adverse events (SAEs) related to sedation in their practices?

Given this background, we conducted a nationwide survey to assess current practices of sedation and concomitant patient monitoring for routine GI endoscopy among Korean endoscopists. Because there is limited evidence on sedation practice patterns among Asian endoscopists, we believe that our data reflecting endoscopists' real-life practices could be a useful source to impact quality improvement in patient care, the provision of proper training programs, and, potentially, policy-making for endoscopic sedation.

\section{MATERIALS AND METHODS}

\section{Study population and design}

This study was a cross-sectional web survey conducted in August 2014 among endoscopists who were active members of the Korean Society for Gastrointestinal Endoscopy (KSGE). All active members of the KSGE who were identified from the KSGE database were invited by email to participate. Survey participants completed an anonymous survey via a dedicated website linked to an email. All completed data were transferred via the web to a central database. All data remained anonymous to study investigators.

\section{Ethical considerations}

This study was conducted according to the Declaration of Helsinki and was approved by the Institutional Review Board of Kyung Hee University Hospital (KMC-IRB 1423-01). The study was registered at the clinical research information service (http:// cris.nih.go.kr/; registration number: KCT0001171). All authors reviewed and approved the final manuscript.

\section{Development and contents of survey items}

A five-section, 35-item questionnaire was developed by expert members of the KSGE Task Force on Endoscopic Sedation (Appendix 1). Briefly, the first section consisted of demographics and basic characteristics of the respondents, including the endoscopist's specialty, years of endoscopic practice, practice environment (primary clinic, nonacademic community hospital, and academic teaching hospital), number of weekly procedures, and type of endoscopic practice (esophagogastroduodenoscopy [EGD] only, colonoscopy only, or both). The second section addressed the endoscopists' dominant sedation method used and their satisfaction with it, rated by 10-point visual analog scale (VAS), and the number of staff present during the sedation practice. The third section was targeted at the responsibility for and actual delivery of propofol and the potential reasons for not using propofol, if indicated. The fourth section regarding monitoring practices addressed the use of preprocedural assessments, using the American Society of Anesthesiologists (ASA) physical status classification, a formal sedation protocol, and patient vigilance, including pulse oximetry and supplemental oxygen. ${ }^{26}$ We also inquired about the endoscopists' experiences with sedation-related SAEs during their practice, predefined as

Table 1. Demographics of Survey Respondents

\begin{tabular}{lc}
\hline \multicolumn{1}{c}{ Characteristic } & Value \\
\hline No. of respondents & $1,332(22.7)$ \\
Male sex & $1,068(80.2)$ \\
Age, yr & $43.4(28.0-80.0)$ \\
Practice environment & \\
Private clinic & $599(45.0)$ \\
Nonacademic hospital & $349(26.2)$ \\
Academic teaching hospital & $372(27.9)$ \\
Others & $12(0.9)$ \\
Specialty & \\
Gastroenterologist & $1,097(82.4)$ \\
General internist & $172(12.9)$ \\
Surgeon & $36(2.7)$ \\
Pediatrician & $23(1.7)$ \\
Others & $4(0.3)$ \\
Years of endoscopy practice & \\
$<5$ & $334(25.1)$ \\
5-10 & $380(28.5)$ \\
11-15 & $222(16.7)$ \\
$>15$ & $231(17.3)$ \\
Type of endoscopic practice & $387(29.1)$ \\
Esophagogastroduodenoscopy & \\
Colonoscopy & $136(10.2)$ \\
Both & \\
No. of endoscopy cases per week & \\
\hline
\end{tabular}

Data are presented as number (\%) or mean (range). 
mask ventilation, endotracheal intubation, permanent injury, or death. The final section addressed training in endoscopic sedation.

\section{Statistical analysis}

We analyzed the data first using descriptive statistics. Then, we performed the chi-square test to assess differences in proportions. Student t-test was used to assess differences between means, such as the endoscopists' satisfaction scores rated by the VAS. Finally, we performed a logistic regression analysis to identify potential predictors of endoscopist experience with sedation-related SAEs, after adjustment for potential confounders, including the respondent's age and gender, specialty, practice environment, type and burden of endoscopic procedures, years of endoscopic practice, dominant sedation method, use of the ASA categories for preprocedural assessment or a formal sedation protocol, use of pulse oximetry or supplemental oxygen, and completion of dedicated sedation training. Factors with a p-value $<0.2$ in the univariate analysis were included in the stepwise regression analysis. A p-value $<0.05$ was considered to indicate statistical significance. All data were analyzed using SPSS version 18.0K for windows (SPSS Korea Inc., Seoul, Korea).

\section{RESULTS}

\section{Characteristics of the study respondents}

The demographic characteristics of the study respondents are summarized in Table 1 . In total, 1,332 of the 5,860 KSGE members invited completed the survey, an overall response rate of $22.7 \%$. The mean age of the respondents was 43.4 years; $80.2 \%$ were men, and $82.4 \%$ were gastroenterologists. Of the respondents, $46 \%$ currently practiced at a primary clinic, $26.2 \%$ at a nonacademic hospital, and 27.9\% at an academic teaching hospital. Of the respondents, $46.4 \%$ had $\geq 10$ years of endoscopic practice, 88\% currently performed both EGD and colonoscopy, and $79.4 \%$ performed $\geq 20$ endoscopies per week.

\section{Dominant sedation method and endoscopists' satisfaction}

The vast majority of respondents (98.9\%, 1,318/1,332) currently offer procedural sedation for diagnostic EGD (99.1\%) and colonoscopy (91.4\%). The detailed proportions of sedation use in EGD and colonoscopy are summarized in Table 2. Propofolbased sedation (propofol alone or in combination with midazolam and/or an opioid) was the most preferred sedation method for both EGD and colonoscopy (55.6\% and 52.6\%, respectively). Regarding endoscopists' satisfaction with their primary sedation method, the mean (standard deviation) satisfaction score for

Table 2. The Use of Sedation in Elective Esophagogastroduodenoscopy and Colonoscopy

\begin{tabular}{|c|c|c|}
\hline Variable & EGD & Colonoscopy \\
\hline Current use of sedation, if any & $1,305(99.0)$ & $1,205(91.4)$ \\
\hline \multicolumn{3}{|l|}{ Proportion of sedated endoscopy } \\
\hline$<25 \%$ of cases & $124(9.5)$ & $19(1.6)$ \\
\hline $26 \%-50 \%$ of cases & $298(22.8)$ & $57(4.7)$ \\
\hline $51 \%-75 \%$ of cases & $474(36.3)$ & $188(15.6)$ \\
\hline$>76 \%$ of cases & 409 (31.3) & $941(78.1)$ \\
\hline \multicolumn{3}{|l|}{ Endoscopists' choice } \\
\hline Midazolam \pm opioid & $483(37.0) / 54(4.1)$ & $185(15.4) / 360(29.9)$ \\
\hline Propofol \pm opioid & $378(29.0) / 2(0.2)$ & $72(6.0) / 13(1.1)$ \\
\hline Propofol+midazolam \pm opioid & $330(25.3) / 15(1.1)$ & $407(33.8) / 143(11.9)$ \\
\hline Others & $43(3.3)$ & $25(2.1)$ \\
\hline \multicolumn{3}{|c|}{ Overall endoscopists' satisfaction with sedation } \\
\hline $9-10$ & $339(26.0)$ & 457 (37.9) \\
\hline $7-8$ & $688(52.7)$ & $577(47.9)$ \\
\hline $5-6$ & $191(14.6)$ & $129(10.7)$ \\
\hline$\leq 4$ & $87(6.7)$ & $42(3.5)$ \\
\hline \multicolumn{3}{|l|}{ Staffing in endoscopic sedation* } \\
\hline One nurse ${ }^{\dagger}$ & \multicolumn{2}{|c|}{$417(31.6)$} \\
\hline Two nurses $^{\dagger}$ & \multicolumn{2}{|c|}{$813(61.7)$} \\
\hline One assisting physician and $\geq 1$ nurse $^{\dagger}$ & \multicolumn{2}{|c|}{$88(6.7)$} \\
\hline
\end{tabular}

Data are presented as number (\%).

EGD, esophagogastroduodenoscopy.

${ }^{*}$ Except for endoscopist; ${ }^{\dagger}$ Trained registered or licensed practical nurse. 
propofol-based sedation was significantly higher than that for standard sedation (7.99 [1.29] vs 6.60 [1.78] for EGD; 8.24 [1.23] vs 7.45 [1.64] for colonoscopy, respectively; all $\mathrm{p}<0.001)$. More than half $(61.7 \%)$ worked with two trained nurses (registered or licensed practical nurses) for sedated endoscopy.

\section{Propofol sedation}

Of the respondents, $63 \%(830 / 1,318)$ of respondents currently used propofol with good satisfaction ratings: 91.1\% rated 7 points or more on a VAS. Use of propofol was almost always

Table 3. Practice Patterns for the Use of Propofol ( $n=830)$

\begin{tabular}{lc}
\hline \multicolumn{1}{c}{ Variable } & No. (\%) \\
\hline Supervision for the use of propofol & $818(98.6)$ \\
By endoscopist & $3(0.4)$ \\
By assisting nonanesthesiologist physician & $2(0.2)$ \\
By anesthesiologist & $7(0.8)$ \\
By endoscopy nurse* & \\
Administration (delivery) of propofol & $80(9.6)$ \\
By endoscopist & $13(1.6)$ \\
By assisting physician & $2(0.2)$ \\
By anesthesiologist & $735(88.5)$ \\
By endoscopy nurse* & \\
Overall satisfaction score & \\
$9-10$ & $336(40.5)$ \\
$7-8$ & $420(50.6)$ \\
$5-6$ & $54(6.5)$ \\
$\leq 4$ & $20(2.4)$ \\
\hline
\end{tabular}

${ }^{*}$ Trained registered or licensed practical nurse; ${ }^{\dagger}$ Measured on a 10-point visual analog scale.

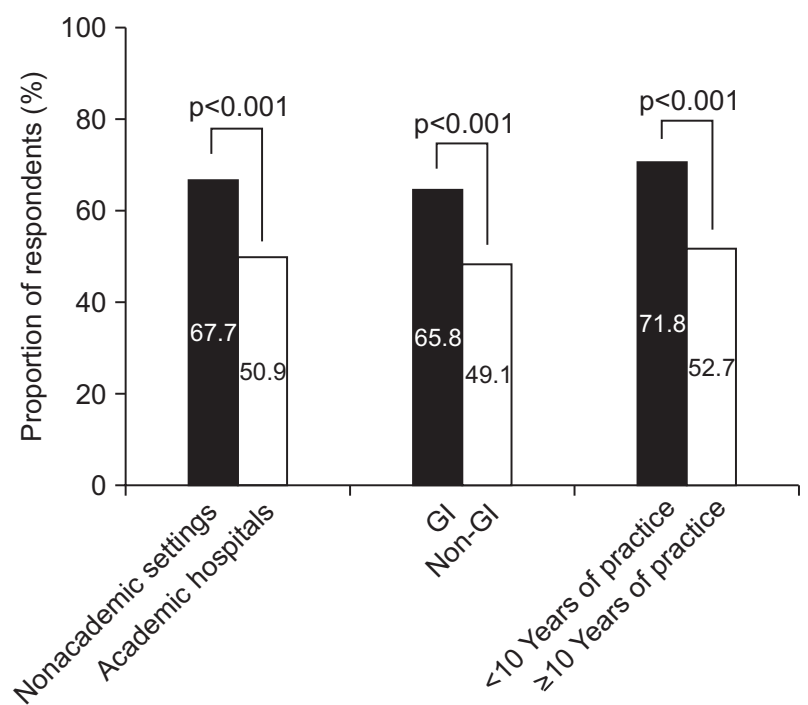

Fig. 1. The characteristics of respondents who chose propofol as the dominant sedation method.

GI, gastrointestinal. directed by endoscopists (98.6\%), but delivery of the drug was performed mostly by trained nurses (88.5\%) (Table 3). Endoscopists practicing in nonacademic settings, gastroenterologists, or endoscopists with $<10$ years of practice were more likely to use propofol than were endoscopists work in an academic hospital, nongastroenterologists, or endoscopists with $\geq 10$ years of practice $(67.7 \%$ vs $50.9 \%, 65.8 \%$ vs $49.1 \%$, and $71.8 \%$ vs $52.7 \%$, respectively; all $\mathrm{p}<0.001$ ) (Fig. 1). The fear of cardiopulmonary side effects was the most common reason cited for not currently using propofol (50.0\%, 244/488).

\section{Concurrent monitoring practices}

The majority of respondents $(94.1 \%, 1,240 / 1,318)$ always or usually monitored pulse oximetry, and 42.5\% (560/1,318) always or usually administered supplemental oxygen during sedated endoscopy (Fig. 2). Compared with their counterparts, endoscopists practicing at an academic hospital, gastroenterologists, and endoscopists with $<10$ years of practice were more likely to use pulse oximetry or supplemental oxygen (all $\mathrm{p}<0.001$ ) (Table 4). Of the respondents, 38.3\% of respondents rarely or never performed risk stratification using the ASA categories prior to providing sedation. Endoscopists practicing at an academic hospital were more likely to use ASA categories than were endoscopists practicing in nonacademic settings (74.7\% vs $56.6 \%, \mathrm{p}<0.001)$, but this finding was not associated with the endoscopists' specialty or years of endoscopic practice. Of the respondents, $27.4 \%(361 / 1,318)$ provided sedatives using no formal sedation protocol. Endoscopists practicing in nonacademic settings and nongastroenterologists were more likely not

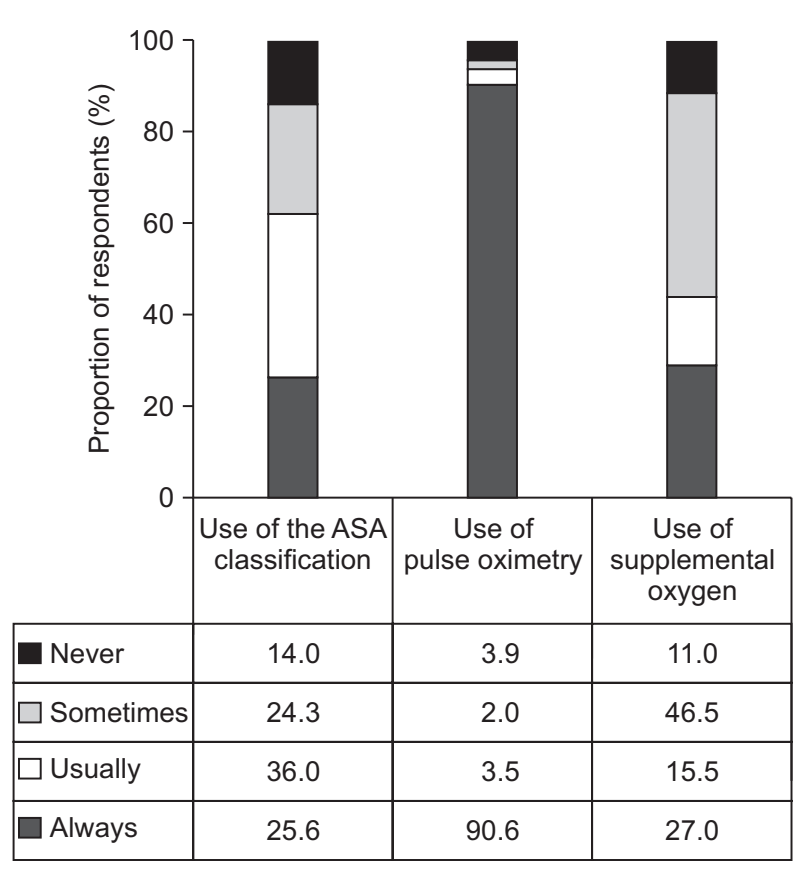

Fig. 2. Practice patterns regarding concomitant patient monitoring. ASA, American Society of Anesthesiologists. 
Table 4. Patient Monitoring Practices

\begin{tabular}{|c|c|c|c|c|c|c|c|c|c|}
\hline & $\begin{array}{c}\text { Nonacademic } \\
\text { settings }\end{array}$ & $\begin{array}{l}\text { Academic } \\
\text { hospitals }\end{array}$ & $\mathrm{p}$-value & GI & Non-GI & $\mathrm{p}$-value & $\begin{array}{l}<10 \text { Years } \\
\text { of practice }\end{array}$ & $\begin{array}{l}\geq 10 \text { Years } \\
\text { of practice }\end{array}$ & p-value \\
\hline ASA category ${ }^{*}, \%$ & 56.6 & 74.8 & $<0.001$ & 62.6 & 57.1 & 0.073 & 59.9 & 63.7 & 0.089 \\
\hline Pulse oximetry ${ }^{*}, 0$ & 91.8 & 100.0 & $<0.001$ & 97.2 & 79.9 & $<0.001$ & 99.3 & 88.0 & $<0.001$ \\
\hline Supplemental oxygen*, \% & 38.6 & 52.6 & $<0.001$ & 44.6 & 32.1 & $<0.001$ & 47.1 & 37.1 & $<0.001$ \\
\hline Sedation protocol & 64.4 & 93.8 & $<0.001$ & 75.1 & 60.3 & $<0.001$ & 72.1 & 73.2 & 0.665 \\
\hline
\end{tabular}

GI, gastrointestinal; ASA, American Society of Anesthesiologists.

*Proportion of respondents who always or usually used.

to use a formal sedation protocol, when compared with their counterparts (35.6\% vs 6.2\%, 39.7\% vs $24.9 \%$, respectively; all $\mathrm{p}<0.001)$.

Regardng SAEs, $65.4 \%(814 / 1,318)$ of respondents stated they had experience with one of the four predefined SAEs during their own life-time practice: mask ventilation in 61.8\%, endotracheal intubation in $16.2 \%$, permanent injury in $1.4 \%$, and death in $1.7 \%$. The choice of propofol as the dominant sedation method was the only significant predictor of endoscopist experience with sedation-related SAEs (odds ratio, 1.854; 95\% confidence interval, 1.414 to $2.432 ; \mathrm{p}<0.001$ ), after adjusting for potential confounders.

\section{Sedation training}

Respondents stated that they received dedicated sedation training at their training hospital (30.1\%) or continuing education programs by professional organizations (18.4\%), or both (42.6\%). However, 8.9\% of all respondents had not received any training or education regarding sedation practices. Among gastroenterologists, 20.1\% (220/1,097) did not receive dedicated training for sedation during their GI fellowship. More than half of respondents (54.3\%) agreed that assessment of competence is required following specialized training or education for endoscopic sedation. Finally, 45.4\% of respondents stated that periodic retraining on basic life support techniques was not enforced for all medical personnel involved in sedation practices within their unit.

\section{DISCUSSION}

This nationwide survey of 1,332 endoscopists demonstrated that endoscopist-directed, nurse-administered propofol is currently the dominant sedation method used in routine GI endoscopy in Korea. Although many previous surveys have reported that "standard" sedation using midazolam and/or opioids still prevails among most Western endoscopists for routine endoscopy, a recent survey in Spain showed that propofol has become increasingly accepted as the dominant sedative in GI endoscopy. ${ }^{18-25}$ In our study, the preference for propofol by endoscopists was associated with the endoscopist's specialty (gastroenterologist), practice environment (nonacademic setting), and years of endoscopic practice ( $<10$ years of practice). These findings are consistent with our expectations to some extent. First, propofol is a highly attractive sedative for endoscopists due to its proven efficacy in GI endoscopy. Previous studies have consistently reported that compared with standard sedation, propofol sedation during routine GI endoscopy provides better patient satisfaction with no increase in adverse events in healthy individuals. ${ }^{1,2,5,6}$ As demonstrated in this survey, propofol sedation also provided better endoscopist satisfaction than did standard sedation, consistent with previous surveys of Western endoscopists. ${ }^{18-25} \mathrm{In}$ addition, the use of propofol for procedural sedation is freely permitted for nonanesthesiologists in Korea. With recent increased use and the subsequent popularity of propofol, training in EDP is more integrated as a basic course during fellowships in gastroenterology for Korean trainees. Thus, we assumed that gastroenterologists who had completed fellowships recently would prefer propofol over standard sedation.

It also seems likely that operating efficiency may be a possible explanation for why propofol was preferred by endoscopists practicing in nonacademic settings, where available resources are expected to be more limited than those at an academic hospital. Therefore, the pharmacokinetics of propofol, such as its rapid onset of action and shorter recovery time, are presumed to be a potential factor influencing an endoscopist's decision on sedative use. In fact, recent meta-analyses have suggested that propofol sedation can affect endoscopy unit efficiency without compromising patient care during GI endoscopy. ${ }^{27,28}$ One survey of endoscopists in the United States reported findings similar to ours. ${ }^{29}$ In that study, community practitioners were more likely to use propofol than those at academic centers, regardless of the procedure (EGD, colonoscopy, or endoscopic ultrasonography/ endoscopic retrograde cholangiopancreatography). ${ }^{29}$ However, there appears to be some regional variation associated with the preference for propofol by Korean endoscopists, which may be due partly to limited resources at anesthesiology services and partly to lower reimbursement for sedation practices.

Appropriate training of endoscopists who are responsible for the administration of sedation is the first step to guarantee patient safety during endoscopic sedation. Thus, current guidelines recommend that healthcare providers who plan to administer sedation should receive proper training in procedural sedation 
prior to the beginning of their own practice., ${ }^{2,630}$ Our study demonstrated that $72.7 \%$ of respondents received dedicated training in endoscopic sedation, whereas $18.4 \%$ received only continuing educational programs by professional organizations, and 8.9\% depended only on self-directed learning. Interestingly, 20.1\% of Korean gastroenterologists did not receive dedicated training in endoscopic sedation during their fellowship. Likewise, a recent study in the United Kingdom reported that 51\% of gastroenterology trainees did not receive structural training in safe sedation. ${ }^{31}$ Those findings, including ours, provide several important suggestions regarding the provision of educational programs for endoscopic sedation. First, a dedicated training course on endoscopic sedation should be provided for trainees in gastroenterology during fellowships. Additionally, professional GI societies should offer more structured educational programs, including hands-on skills, for endoscopists who are not trained in endoscopic sedation, given that continuous educational programs generally consist of didactic lectures only. In this study, more than half of respondents (54.3\%) stated that an assessment of competence should be required following specialized training or education for endoscopic sedation. These attitudes are aligned with current guidelines recommending assessment of competency following completion of training courses. ${ }^{32,33}$

Our study also raises other sedation quality issues related to patient vigilance. First, a substantial proportion of respondents (38.3\%) stated that they had never or only sometimes used the ASA classification for preprocedural assessment. An anesthesiology service is generally recommended for endoscopic sedation of patients with a higher ASA category who are believed to have definite patient-related risk factors for complications., ${ }^{2,6,30}$ Thus, stratification by established methods, such as ASA category, is now recommended as an important quality indicator for all GI endoscopic procedures. ${ }^{34}$ In addition, 27.4\% of respondents stated that they performed sedation practice with no formal sedation protocol. Even if sedation practice is highly variable according to endoscopists, the use of a formal sedation protocol is required for standardization of patient care. In this regard, a recent study conducted in Germany provides important evidence suggesting that quality can be improved significantly by implementation of appropriate quality measures. ${ }^{35}$ In a study surveying 4,405 members of the German Society of Digestive and Metabolic Diseases, patient monitoring practices during endoscopic sedation were clearly improved by the 3-year implementation of national sedation guidelines for GI endoscopy. ${ }^{35}$ We believe that our study provides a rationale for the development of national guidelines on sedation during GI endoscopy.

One of several interesting findings in this study is that a large number of endoscopists have experience in serious respiratory depression requiring airway rescue in their own practice (mask ventilation in 61.8\%, endotracheal intubation in 16.2\%). Respiratory depression is the most common serious, potentially life-threatening complication related to sedation, regardless of the type of sedative or analgesic. ${ }^{2,30}$ Despite numerous studies demonstrating the safety of endoscopic sedation, especially EDP, most results were obtained from randomized controlled trials or large nonrandomized case series, rather than a reallife clinical setting. ${ }^{1,6-10,27,28}$ In this regard, our current findings suggest that the incidence of serious respiratory events during endoscopic sedation is underestimated in real-life settings. In fact, the proportion of endoscopists who had experience in serious respiratory events in this study was much higher than our expectations. Although we could not quantify the usual depth of sedation, we assumed that most endoscopists might use moderate sedation, because our survey focused on elective EGD and colonoscopy. In addition, the reported rates of pulse oximetry (94.1\%) and supplemental oxygen (42.5\%) use in the present study are generally comparable with those of previous sedation surveys reported in Europe and North America, ranging from $77 \%$ to $100 \%$ for pulse oximetry use and from $34.0 \%$ to $72.7 \%$ for supplemental oxygen use. ${ }^{18-25}$ Ultimately, our findings support the importance of airway management in the provision of educational programs for sedation training. We also believe that all medical personnel involved in endoscopic sedation should undergo periodic retraining in airway management, given that actual delivery of the sedatives was performed mostly by endoscopy nurses in this study.

The use of propofol as a preferred sedation method was the only significant predictor of endoscopist experience with sedation-related SAEs in the current study. However, this finding should be interpreted carefully, given that the survey was not intended to calculate the actual incidences of adverse events related to propofol-based sedation. As stated above, numerous studies have consistently shown that propofol sedation presents similar rates of adverse events, provides higher patient and physician satisfaction, and decreases procedure and recovery times, when compared with standard sedation. ${ }^{1,2,5,6,27,28}$ In addition, there is accumulating evidence that EDP can be used safely and effectively for healthy individuals in elective GI endoscopy ${ }^{6-10,27,28}$ A recent large prospective study evaluating 10,000 patients who underwent endoscopic procedures also confirmed that EDP in an outpatient setting is a safe procedure among patients without severe comorbidities. ${ }^{9}$ Given the different practice settings among countries, we believe that a future prospective cohort study is required to evaluate the safety of EDP in Korea.

This study has several limitations. First, it was a cross-sectional analysis using a self-reported survey, so recall bias could be an issue. Although we maintained the anonymity of respondents, further prospective cohort studies are required to deal with sensitive issues such as the endoscopist's experience with SAEs related to sedation in real-life settings. Second, the final response rate was low (22.7\%), so it may be argued that our data are not representative of the entire population of Korean endoscopists. Given the large number of respondents who com- 
pleted the survey, however, we believe that our data adequately reflect current sedation practice in Korea. Finally, we did not survey some important quality issues in endoscopic sedation, such as informed consent and documentation of sedation practices. ${ }^{33}$ In a study analyzing anesthesia-related medical disputes during a 6-year period in Korea, lack of records on evaluation pre- and during anesthesia were reported in 92.3\% and 89.7\%, respectively, among 39 sedation cases with medical disputes. ${ }^{13}$ A recently published guideline for quality indicators common to all GI endoscopic procedures also highlights that formal documentation is essential for the entire endoscopic sedation process, including a sedation plan, risk assessment of patients before sedation, patient monitoring during sedation, and the use of reversal agents if indicated. ${ }^{34}$ We believe that this is an important topic for quality improvement in endoscopic sedation.

In conclusion, the present nationwide study reveals that endoscopist-directed, nurse-administered propofol has become standard practice for endoscopic sedation in Korea. The preference for propofol is currently headed by endoscopists practicing in nonacademic settings, gastroenterologists, or endoscopists with $<10$ years of endoscopic practice. However, this study strongly suggests there is much room for quality improvement in endoscopist-directed sedation, especially in sedation training and patient vigilance. Given the current results regarding sedation-related SAEs, we believe that expertise in airway rescue techniques should become a key priority in training and continuing educational programs for endoscopic sedation.

\section{CONFLICTS OF INTEREST}

No potential conflict of interest relevant to this article was reported.

\section{ACKNOWLEDGEMENTS}

This study was supported by a 2014 DONG-A ST grant from the Korean Gastrointestinal Endoscopy Research Foundation.

\section{REFERENCES}

1. McQuaid KR, Laine L. A systematic review and meta-analysis of randomized, controlled trials of moderate sedation for routine endoscopic procedures. Gastrointest Endosc 2008;67:910-923.

2. Cohen LB, Delegge MH, Aisenberg J, et al. AGA Institute review of endoscopic sedation. Gastroenterology 2007;133:675-701.

3. Nonaka M, Gotoda T, Kusano C, et al. Safety of gastroenterologistguided sedation with propofol for upper gastrointestinal therapeutic endoscopy in elderly patients compared with younger patients. Gut Liver 2015;9:38-42.

4. Kim SG. The elderly also deserves to undergo therapeutic endoscopy safely under sedation with propofol by gastroenterologists. Gut Liver 2015;9:1-2.
5. Lazzaroni M, Bianchi Porro G. Preparation, premedication, and surveillance. Endoscopy 2001;33:103-108.

6. Vargo JJ, Cohen LB, Rex DK, Kwo PY. Position statement: nonanesthesiologist administration of propofol for GI endoscopy. Am J Gastroenterol 2009;104:2886-2892.

7. Rex DK, Deenadayalu VP, Eid E, et al. Endoscopist-directed administration of propofol: a worldwide safety experience. Gastroenterology 2009;137:1229-1237.

8. Sieg A; bng-Study-Group, Beck S, et al. Safety analysis of endoscopist-directed propofol sedation: a prospective, national multicenter study of 24441 patients in German outpatient practices. J Gastroenterol Hepatol 2014;29:517-523.

9. Friedrich K, Stremmel W, Sieg A. Endoscopist-administered propofol sedation is safe: a prospective evaluation of 10,000 patients in an outpatient practice. J Gastrointestin Liver Dis 2012;21:259263.

10. Behrens A, Labenz J, Schuler A, et al. How safe is sedation in gastrointestinal endoscopy? A multicentre analysis of 388,404 endoscopies and analysis of data from prospective registries of complications managed by members of the Working Group of Leading Hospital Gastroenterologists (ALGK). Z Gastroenterol 2013;51:432436.

11. Perel A. Non-anaesthesiologists should not be allowed to administer propofol for procedural sedation: a Consensus Statement of 21 European National Societies of Anaesthesia. Eur J Anaesthesiol 2011;28:580-584.

12. Pelosi P; Board of the European Society of Anaesthesiology. Retraction of endorsement: European Society of Gastrointestinal Endoscopy, European Society of Gastroenterology and Endoscopy Nurses and Associates and the European Society of Anaesthesiology Guideline: non-anaesthesiologist administration of propofol for gastrointestinal endoscopy. Eur J Anaesthesiol 2012;29:208.

13. Roh WS, Kim DK, Jeon YH, et al. Analysis of anesthesia-related medical disputes in the 2009-2014 period using the Korean society of anesthesiologists database. J Korean Med Sci 2015;30:207-213.

14. Hassan C, Rex DK, Cooper GS, Benamouzig R. Endoscopist-directed propofol administration versus anesthesiologist assistance for colorectal cancer screening: a cost-effectiveness analysis. Endoscopy 2012;44:456-464.

15. Liu H, Waxman DA, Main R, Mattke S. Utilization of anesthesia services during outpatient endoscopies and colonoscopies and associated spending in 2003-2009. JAMA 2012;307:1178-1184.

16. Khiani VS, Soulos P, Gancayco J, Gross CP. Anesthesiologist involvement in screening colonoscopy: temporal trends and cost implications in the medicare population. Clin Gastroenterol Hepatol 2012;10:58-64.e1.

17. Triantafillidis JK, Merikas E, Nikolakis D, Papalois AE. Sedation in gastrointestinal endoscopy: current issues. World J Gastroenterol 2013;19:463-481.

18. Heuss LT, Froehlich F, Beglinger C. Changing patterns of sedation and monitoring practice during endoscopy: results of a nationwide survey in Switzerland. Endoscopy 2005;37:161-166. 
19. Ladas SD, Aabakken L, Rey JF, et al. Use of sedation for routine diagnostic upper gastrointestinal endoscopy: a European Society of Gastrointestinal Endoscopy Survey of National Endoscopy Society Members. Digestion 2006;74:69-77.

20. Cohen LB, Wecsler JS, Gaetano JN, et al. Endoscopic sedation in the United States: results from a nationwide survey. Am J Gastroenterol 2006;101:967-974.

21. Paspatis GA, Manolaraki MM, Tribonias G, et al. Endoscopic sedation in Greece: results from a nationwide survey for the Hellenic Foundation of gastroenterology and nutrition. Dig Liver Dis 2009;41:807-811.

22. Riphaus A, Rabofski M, Wehrmann T. Endoscopic sedation and monitoring practice in Germany: results from the first nationwide survey. Z Gastroenterol 2010;48:392-397.

23. Porostocky P, Chiba N, Colacino P, Sadowski D, Singh H. A survey of sedation practices for colonoscopy in Canada. Can J Gastroenterol 2011;25:255-260.

24. Fanti L, Agostoni M, Gemma M, et al. Sedation and monitoring for gastrointestinal endoscopy: a nationwide web survey in Italy. Dig Liver Dis 2011;43:726-730.

25. Lucendo AJ, González-Huix F, Tenias JM, et al. Gastrointestinal endoscopy sedation and monitoring practices in Spain: a nationwide survey in the year 2014. Endoscopy 2015;47:383-390.

26. Dripps RD, Lamont A, Eckenhoff JE. The role of anesthesia in surgical mortality. JAMA 1961;178:261-266.

27. Wang D, Chen C, Chen J, et al. The use of propofol as a sedative agent in gastrointestinal endoscopy: a meta-analysis. PLoS One 2013;8:e53311.

28. Sethi S, Wadhwa V, Thaker A, et al. Propofol versus traditional sedative agents for advanced endoscopic procedures: a metaanalysis. Dig Endosc 2014;26:515-524.

29. Faulx AL, Vela S, Das A, et al. The changing landscape of practice patterns regarding unsedated endoscopy and propofol use: a national Web survey. Gastrointest Endosc 2005;62:9-15.

30. Standards of Practice Committee of the American Society for Gastrointestinal Endoscopy, Lichtenstein DR, Jagannath S, et al. Sedation and anesthesia in GI endoscopy. Gastrointest Endosc 2008;68:815-826.

31. Mohanaruban A, Bryce K, Radhakrishnan A, Gallaher J, Johnson G. Safe sedation practices among gastroenterology registrars: do we need more training? Frontline Gastroenterol 2015;6:223-228.

32. Dumonceau JM, Riphaus A, Beilenhoff U, et al. European curriculum for sedation training in gastrointestinal endoscopy: position statement of the European Society of Gastrointestinal Endoscopy (ESGE) and European Society of Gastroenterology and Endoscopy Nurses and Associates (ESGENA). Endoscopy 2013;45:496-504.

33. American Association for Study of Liver Diseases; American College of Gastroenterology; American Gastroenterological Association Institute, et al. Multisociety sedation curriculum for gastrointestinal endoscopy. Gastrointest Endosc 2012;76:e1-e25.

34. Rizk MK, Sawhney MS, Cohen J, et al. Quality indicators common to all GI endoscopic procedures. Gastrointest Endosc 2015;81:316.

35. Riphaus A, Geist F, Wehrmann T. Endoscopic sedation and monitoring practice in Germany: re-evaluation from the first nationwide survey 3 years after the implementation of an evidence and consent based national guideline. Z Gastroenterol 2013;51:10821088. 
Appendix 1. Sedation Survey Questionnaire

\section{Part I. Demographics of survey respondents}

1) Sex

(1) Male

(2) Female

2) Age: ( ) years

3) Practice environment

(1) Primary clinic

(2) Nonacademic hospital

(3) Academic teaching hospital

(4) Others

4) What is your specialty?

(1) Gastroenterologist

(2) General internist

(3) Surgeon

(4) Pediatrician

(5) Others

5) How long have you performed endoscopy (years of endoscopic practice)?
(1) <5 years
(2) 5-10 years
(3) 10-15 years
(4) >15 years

6) What kind of endoscopy do you currently perform in your practice?

(1) Esophagogastroduodenoscopy (EGD)

(2) Colonoscopy

(3) Both

7) How many endoscopy cases do you usually perform in your practice?
(1) $<20$ cases
(2) 20-50 cases
(3) 50-100 cases
(4) $>100$ cases

8) Do you currently provide procedural sedation for elective gastrointestinal (GI) endoscopy (EGD or colonoscopy)?

(1) Yes

(2) No ( $\rightarrow$ Jump to question 32)

\section{Part II. Use of sedation in elective EGD and colonoscopy}

9) Do you currently offer sedation for EGD?
(1) Yes
(2) No ( $\rightarrow$ Jump to question 13) 
92 Gut and Liver, Vol. 10, No. 1, January 2016

10) How often do you offer sedation for EGD?

(1) $<25 \%$ of cases

(2) $26 \%-50 \%$ of cases

(3) $51 \%-75 \%$ of cases

(4) $>76 \%$ of cases

11) What kind of sedative(s) is (are) the drug(s) that you prefer for sedated EGD?

(1) Midazolam \pm opioid

(2) Propofol \pm opioid

(3) Propofol+midazolam \pm opioid

(4) Others

12) Rate your overall satisfaction with the sedative(s) that you prefer for sedated EGD, using a 10-point scale (0: unsatisfactory, 10: very satisfied): $(0,1,2,3,4,5,6,7,8,9,10)$.

13) Do you currently offer sedation for colonoscopy?

(1) Yes

(2) No (Jump to question 17)

14) How often do you offer sedation for colonoscopy?

(1) $<25 \%$ of cases

(2) $26 \%-50 \%$ of cases

(3) $51 \%-75 \%$ of cases

(4) $>76 \%$ of cases

15) What kind of sedative(s) is (are) the drug(s) that you prefer for sedated colonoscopy?

(1) Midazolam \pm opioid

(2) Propofol \pm opioid

(3) Propofol+midazolam \pm opioid

(4) Others

16) Rate your overall satisfaction with the sedative(s) that you prefer for sedated colonoscopy, using a 10-point scale (0: unsatisfactory, 10: very satisfied): $(0,1,2,3,4,5,6,7,8,9,10)$.

17) Who is responsible for administration of sedative(s) in your endoscopic practice?

(1) Endoscopist

(2) Assisting nonanesthesiologist physician

(3) Anesthesiologist

(4) Trained nurse (regular nurse or licensed practical nurse)

18) Who actually delivers the sedative(s) to patients in your endoscopic practice?

(1) Endoscopist

(2) Assisting nonanesthesiologist physician

(3) Anesthesiologist

(4) Trained nurse (regular nurse or licensed practical nurse) 
19) How many staffs are involved in your sedation practice (other than the endoscopist)?

(1) One trained nurse

(2) Two trained nurses

(3) One assisting physician and one or more nurses

\section{Part III. Practice patterns for the use of propofol}

20) Do you currently use propofol in your sedation practice?

(1) Yes

(2) No ( $\rightarrow$ Jump to question 24)

21) Who is responsible for the administration of propofol in your endoscopic practice?

(1) Endoscopist

(2) Assisting nonanesthesiologist physician

(3) Anesthesiologist

(4) Trained nurse (regular nurse or licensed practical nurse)

22) Who actually delivers propofol to patients in your endoscopic practice?

(1) Endoscopist

(2) Assisting nonanesthesiologist physician

(3) Anesthesiologist

(4) Trained nurse (regular nurse or licensed practical nurse)

23) Rate your overall satisfaction with propofol sedation, using 10-point scale (0: unsatisfactory, 10: very satisfied): $(0,1,2,3,4,5,6,7,8,9,10)$.

24) What is the most important reason for not currently using propofol for sedated endoscopy?

(1) No need to use propofol

(2) Concerns regarding propofol-related adverse events

(3) Shortage of manpower or lack of facilities

(4) Regulation by the health authorities

(5) Lack of education on and/or experience with propofol sedation

(6) Others

\section{Part IV. Concomitant monitoring practices}

25) How often do you assess your patients using the American Society of Anesthesiologists (ASA) physical status classification, prior to providing sedation?
(1) Always
(2) Usually
(3) Sometimes
(4) Never

26) How often do you monitor oxygen saturation using pulse oximetry during sedated endoscopy?
(1) Always
(2) Usually
(3) Sometimes
(4) Never 
94 Gut and Liver, Vol. 10, No. 1, January 2016

27) How often do you provide supplemental oxygen during sedated endoscopy?
(1) Always
(2) Usually
(3) Sometimes
(4) Never

28) Are you equipped with an emergency cardiopulmonary resuscitation kit within your endoscopy unit?
(1) Yes
(2) No

29) Do you provide sedation using a formal sedation protocol?

(1) Yes

(2) No

30) Do you have any experience in sedation-related serious adverse events in your own endoscopic practice? (Indicate all of the following, if any)

(1) Mask ventilation (associated with serious respiratory depression)

(2) Endotracheal intubation (associated with serious respiratory depression)

(3) Permanent injury

(4) Death

\section{Part V. Sedation training}

31) Have you ever received training or education for sedation practice?

(1) Yes: a dedicated sedation training in a training hospital

(2) Yes: educational programs by professional organizations

(3) Yes: both of above

(4) None

32) Is periodic retraining of basic life support offered to all medical personnel who are involved in sedation practice within your endoscopy unit?
(1) Yes
(2) No

33) Do you think that competency assessment is required following completion of training or education for sedation practice?

(1) Yes

(2) No

34) Do you think that endoscopist-directed propofol sedation is safe and effective for healthy individuals during elective GI endoscopy?
(1) Yes
(2) No

35) Do you think that a national guideline for endoscopic sedation is required?

(1) Yes

(2) No 